\title{
Soliton and Breather Solutions for the Mixed Nonlinear Schrödinger Equation via N-Fold Darboux Transformation
}

\author{
Hui-Qin Hao, Jian-Wen Zhang, and Rui Guo \\ School of Mathematics, Taiyuan University of Technology, Taiyuan 030024, China \\ Correspondence should be addressed to Jian-Wen Zhang; zjwtyut@126.com \\ Received 19 February 2014; Accepted 29 April 2014; Published 21 May 2014 \\ Academic Editor: Abdul Hamid Kara
}

Copyright (C) 2014 Hui-Qin Hao et al. This is an open access article distributed under the Creative Commons Attribution License, which permits unrestricted use, distribution, and reproduction in any medium, provided the original work is properly cited.

Under investigation in this paper is the mixed nonlinear Schrödinger equation, which describes the propagation of the subpicosecond or femtosecond optical pulse in a monomodal optical fiber. The Darboux transformation is constructed and $N$ times iterative potential formula is presented. Two-soliton and breather solutions are derived on the vanishing and two types of nonvanishing backgrounds: the continuous wave $(\mathrm{cw})$ background and constant background, respectively. The dynamic features of the solitons and breathers are discussed via analytic solutions and graphical illustration.

\section{Introduction}

Investigations on the nonlinear Schrödinger equation have attracted certain interest in nonlinear optics [1-6]. In this paper, we mainly investigate the following mixed nonlinear Schrödinger equation (MNLS) [7]:

$$
i u_{z}+\frac{1}{2} u_{t t}+\sigma \frac{1}{4}|u|^{2} u-\sigma \frac{3 i \delta}{16}\left(|u|^{2} u\right)_{t}+\Gamma u=0,
$$

where $u$ denotes the slowly varying complex pulse envelope, $z$ and $t$ are the partial derivatives with respect to the longitudinal distance and retarded time, $\sigma= \pm 1$ corresponds to the focusing and defocusing nonlinear Schrödinger (NLS) equation, $\delta$ is a real parameter describing the measure of the derivative cubic nonlinearity, and $\Gamma$ denotes the loss or gain coefficient. Equation (1) usually governs the subpicosecond or femtosecond optical pulse propagation in a monomodal optical fiber.

As a complete integrability model, (1) is equivalent to the following Wadati-Konno-Ichikawa (WKI) spectral system $[7,8]$ :

$$
\begin{aligned}
& \Psi_{t}=U \Psi=\left(\begin{array}{cc}
\frac{2 i\left(\lambda^{2}-1\right)}{3 \delta} & \frac{i}{2} \lambda u \\
\frac{i}{2} \lambda u^{*} & \frac{2 i\left(\lambda^{2}-1\right)}{3 \delta}
\end{array}\right) \Psi \\
& \Psi_{z}=V \Psi=\left(\begin{array}{cc}
-\frac{4 i}{9 \delta^{2}}\left(\lambda^{2}-1\right)^{2}+\frac{i}{8} \lambda^{2}|u|^{2}+i \frac{\Gamma}{2} & -\frac{i \lambda\left(\lambda^{2}-1\right)}{3 \delta} u+\frac{3 i}{32} \delta \lambda|u|^{2} u-\frac{\lambda}{4} u_{t} \\
-\frac{i \lambda\left(\lambda^{2}-1\right)}{3 \delta} u^{*}+\frac{3 i}{32} \delta \lambda|u|^{2} u^{*}+\frac{\lambda}{4} u_{t}^{*} & \frac{4 i}{9 \delta^{2}}\left(\lambda^{2}-1\right)^{2}-\frac{i}{8} \lambda^{2}|u|^{2}-i \frac{\Gamma}{2}
\end{array}\right) \Psi,
\end{aligned}
$$


where $\Psi=\left(\psi_{1}, \psi_{2}\right)^{\prime}\left({ }^{\prime}\right.$ denotes the transpose of a matrix). One can deduce (1) directly from the zero curvature equation $U_{z}-V_{t}+U V-V U=0$.

During the past decades, [8-10] have derived some soliton solutions via the dressing method, the Hirota bilinear method and the technique of determinant calculation, respectively. However, to our knowledge, the soliton and breather solutions of (1) have not been generated through $N$ fold Darboux transformation and the interaction characters between two breathers have not been analysed. The aim of this paper is mainly to construct $N$-fold Darboux transformation (DT) in Section 2, derive two-soliton, one- and two-breather solutions via the obtained DT, and discuss dynamic features of those solitons and breathers in Section 3. Finally, our conclusions will be addressed in Section 4.

\section{N-Fold Darboux Transformation}

Taking the transformation as

$$
\begin{aligned}
\Psi^{\prime} & =T \Psi=\left(\begin{array}{ll}
A & B \\
C & D
\end{array}\right) \Psi \\
& =\left(\begin{array}{cc}
(-1)^{N}+\sum_{n=1}^{N} a_{2 n} \lambda^{2 n} & \sum_{n=1}^{N} b_{2 n-1} \lambda^{2 n-1} \\
\sum_{n=1}^{N} c_{2 n-1} \lambda^{2 n-1} & (-1)^{N}+\sum_{n=1}^{N} d_{2 n} \lambda^{2 n}
\end{array}\right) \Psi,
\end{aligned}
$$

we require that $\Psi^{\prime}$ must satisfy

$$
\begin{aligned}
& \Psi_{t}^{\prime}=U^{\prime} \Psi^{\prime}=\left(T_{t}+T U\right) T^{-1} \Psi^{\prime}, \\
& \Psi_{z}^{\prime}=V^{\prime} \Psi^{\prime}=\left(T_{z}+T V\right) T^{-1} \Psi^{\prime},
\end{aligned}
$$

where $U^{\prime}, V^{\prime}$ have the same form as $U, V$. From the rules of the Darboux transformation [11-14], we know that $\left.T\right|_{\lambda=\lambda_{1}} \Psi=$ $0, a_{2 n}, b_{2 n-1}, c_{2 n-1}$, and $d_{2 n}$ can be calculated by the linear algebraic system:

$$
\begin{gathered}
\sum_{n=1}^{N}\left(a_{2 n} \lambda_{j}+\alpha_{j} b_{2 n-1}\right) \lambda_{j}^{2 n-1}=(-1)^{N}, \\
\sum_{n=1}^{N}\left(c_{2 n-1}+\alpha_{j} d_{2 n} \lambda_{j}\right) \lambda_{j}^{2 n-1}=(-1)^{N+1} \alpha_{j},
\end{gathered}
$$

with

$$
\alpha_{j}=\frac{\phi_{2}\left(\lambda_{j}\right)-\gamma_{j} \psi_{2}\left(\lambda_{j}\right)}{\phi_{1}\left(\lambda_{j}\right)-\gamma_{j} \psi_{1}\left(\lambda_{j}\right)}, \quad 1 \leq j \leq 2 N,
$$

and $\phi=\left(\phi_{1}, \phi_{2}\right)^{\prime}, \psi=\left(\psi_{1}, \psi_{2}\right)^{\prime}$ as two basic solutions of the spectral system (2a)-(2b) and $\lambda_{j}$ and $\gamma_{j}\left(\lambda_{k} \neq \lambda_{j}\right.$, $\gamma_{k} \neq \gamma_{j}$, as $k \neq j$ ) as parameters suitably chosen such that the determinant of coefficients for (5) is nonzero. Thus, $a_{2 n}, b_{2 n-1}$, $c_{2 n-1}$, and $d_{2 n}$ are uniquely determined by (5). Equations (3) and (5) show that $\operatorname{det} T(\lambda)$ is a $2 N$ polynomial of $\lambda$, and

$$
\begin{aligned}
& \operatorname{det} T\left(\lambda_{j}\right)=A\left(\lambda_{j}\right) D\left(\lambda_{j}\right)-B\left(\lambda_{j}\right) C\left(\lambda_{j}\right), \\
& A\left(\lambda_{j}\right)=-\alpha_{j} B\left(\lambda_{j}\right), \quad C\left(\lambda_{j}\right)=-\alpha_{j} D\left(\lambda_{j}\right) .
\end{aligned}
$$

Therefore, one can derive that $\operatorname{det} T(\lambda)=\prod_{j=1}^{2 N}\left(\lambda-\lambda_{j}\right)=0$, so $\lambda_{j}(j=1,2, \ldots)$ are $2 N$ roots for the determinant of matrix $T(\lambda)$.

Meanwhile, defining

$$
\begin{aligned}
& \left(T_{t}+T U\right) T^{*}=\left(\begin{array}{ll}
f_{11}(\lambda) & f_{12}(\lambda) \\
f_{21}(\lambda) & f_{22}(\lambda)
\end{array}\right), \\
& \left(T_{z}+T V\right) T^{*}=\left(\begin{array}{ll}
g_{11}(\lambda) & g_{12}(\lambda) \\
g_{21}(\lambda) & g_{22}(\lambda)
\end{array}\right),
\end{aligned}
$$

one can deduce that $f_{11}(\lambda)$ and $f_{22}(\lambda)$ are $(4 N+2), f_{12}(\lambda)$ and $f_{21}(\lambda)$ are $4 N+1, g_{11}(\lambda)$ and $g_{22}(\lambda)$ are $(4 N+4), g_{12}(\lambda)$ and $g_{21}(\lambda)$ are $4 N+3$ th-order polynomials of $\lambda$, and

$$
\begin{gathered}
\alpha_{j t}=\frac{i}{2} \lambda_{j} u^{*}-\frac{4 i\left(\lambda_{j}^{2}-1\right)}{3 \delta} \alpha_{j}-\frac{i}{2} \lambda_{j} u \alpha_{j}^{2}, \\
\alpha_{j z}=c\left(\lambda_{j}\right)-2 a\left(\lambda_{j}\right) \alpha_{j}-b\left(\lambda_{j}\right) \alpha_{j}^{2},
\end{gathered}
$$

where

$$
\begin{gathered}
a\left(\lambda_{j}\right)=-\frac{4 i}{9 \delta^{2}}\left(\lambda_{j}^{2}-1\right)^{2}+\frac{i}{8} \lambda_{j}^{2}|u|^{2}, \\
b\left(\lambda_{j}\right)=-\frac{i \lambda_{j}\left(\lambda_{j}^{2}-1\right)}{3 \delta} u+\frac{3 i}{32} \delta \lambda_{j}|u|^{2} u-\frac{\lambda_{j}}{4} u_{x}, \\
c\left(\lambda_{j}\right)=-\frac{i \lambda_{j}\left(\lambda_{j}^{2}-1\right)}{3 \delta} u^{*}+\frac{3 i}{32} \delta \lambda_{j}|u|^{2} u^{*}+\frac{\lambda_{j}}{4} u_{x}^{*} .
\end{gathered}
$$

By virtue of the above conclusions, one can deduce that all $\lambda_{j}(1 \leqslant j \leqslant 2 N)$ are roots of $f_{k j}(\lambda), g_{k j}(\lambda)(k, j=1,2)$. Noting (7), one can arrive at

$$
\operatorname{det} M\left|f_{k j}(\lambda), \quad \operatorname{det} M\right| g_{k j}(\lambda) \quad(k, j=1,2) .
$$

According to $T T^{*}=\operatorname{det}|T| E$, one can transform (8) into

$$
T_{t}+T U=P(\lambda) T, \quad T_{z}+T V=Q(\lambda) T,
$$

where

$$
\begin{gathered}
P(\lambda)=\left(\begin{array}{cc}
p_{11}^{(2)} \lambda^{2}+p_{11}^{(1)} \lambda+p_{11}^{(0)} & p_{12}^{(1)} \lambda+p_{12}^{(0)} \\
p_{21}^{(1)} \lambda+p_{21}^{(0)} & p_{22}^{(2)} \lambda^{2}+p_{22}^{(1)} \lambda+p_{22}^{(0)}
\end{array}\right), \\
Q(\lambda)=\left(\begin{array}{cc}
\sum_{j=0}^{4} q_{11}^{(j)} \lambda^{j} & \sum_{j=0}^{3} q_{12}^{(j)} \lambda^{j} \\
\sum_{j=0}^{3} q_{21}^{(j)} \lambda^{j} & \sum_{j=0}^{4} q_{22}^{(j)} \lambda^{j}
\end{array}\right)
\end{gathered}
$$


Comparing the coefficients of $\lambda^{2 N+4}, \lambda^{2 N+3}, \lambda^{2 N+2}, \lambda^{2 N+1}$, and $\lambda^{2 N}$ in (12), one can obtain

$$
\begin{aligned}
& p_{11}^{(2)}=-p_{11}^{(0)}=-p_{22}^{(2)}=p_{22}^{(0)}=\frac{2 i}{3 \delta}, \\
& p_{11}^{(1)}=p_{12}^{(0)}=p_{21}^{(0)}=p_{22}^{(1)}=0, \\
& p_{12}^{(1)}=\frac{i}{2}\left(u \frac{a_{2 N}}{d_{2 N}}-\frac{8}{3 \delta} \frac{b_{2 N-1}}{d_{2 N}}\right)=\frac{i}{2} u^{\prime}, \\
& p_{21}^{(1)}=\frac{i}{2}\left(u^{*} \frac{d_{2 N}}{a_{2 N}}+\frac{8}{3 \delta} \frac{c_{2 N-1}}{a_{2 N}}\right)=\frac{i}{2} u^{\prime *} \text {, } \\
& q_{22}^{(4)}=q_{22}^{(0)}=-q_{11}^{(4)}=-q_{11}^{(0)}=\frac{4 i}{9 \delta^{2}}, \\
& q_{11}^{(3)}=q_{11}^{(1)}=q_{12}^{(2)}=q_{12}^{(0)}=q_{21}^{(2)}=q_{21}^{(0)}=q_{22}^{(3)}=q_{22}^{(1)}=0 \text {, } \\
& q_{12}^{(3)}=-\frac{i}{3 \delta}\left(u \frac{a_{2 N}}{d_{2 N}}-\frac{8}{3 \delta} \frac{b_{2 N-1}}{d_{2 N}}\right)=-\frac{i}{3 \delta} u^{\prime}, \\
& q_{21}^{(3)}=-\frac{i}{3 \delta}\left(u^{*} \frac{d_{2 N}}{a_{2 N}}+\frac{8}{3 \delta} \frac{c_{2 N-1}}{a_{2 N}}\right)=-\frac{i}{3 \delta} u^{\prime *}, \\
& q_{11}^{(2)}=\frac{8 i}{9 \delta^{2}}-\frac{i}{3 \delta} \frac{b_{2 N-1}}{a_{2 N}} u^{*}+\frac{i}{8} u u^{*}+q_{12}^{(3)} \frac{c_{2 N-1}}{a_{2 N}} \\
& =\frac{8 i}{9 \delta^{2}}+\frac{i}{8} u^{\prime} u^{\prime *}, \\
& q_{22}^{(2)}=\frac{-8 i}{9 \delta^{2}}+\frac{i}{3 \delta} \frac{c_{2 N-1}}{a_{2 N}} u^{*}-\frac{i}{8} u u^{*}-q_{21}^{(3)} \frac{b_{2 N-1}}{a_{2 N}} \\
& =-\frac{8 i}{9 \delta^{2}}-\frac{i}{8} u^{\prime} u^{\prime *}, \\
& q_{12}^{(1)}=\frac{1}{d_{2 N}}\left(-\frac{8 i}{9 \delta^{2}} b_{2 N-1}+\frac{8 i}{9 \delta^{2}} b_{2 N-3}-q_{11}^{(2)} b_{2 N-1}\right. \\
& -q_{12}^{(3)} d_{2 N-2}+\frac{i u}{3 \delta} a_{2 N}-\frac{i u}{3 \delta} a_{2 N-2}-\frac{i|u|^{2}}{8} b_{2 N-1} \\
& \left.+\frac{3 i \delta}{32} a_{2 N}|u|^{2} u-\frac{u_{t}}{4} a_{2 N}\right) \text {, } \\
& q_{21}^{(1)}=\frac{1}{a_{2 N}}\left(\frac{8 i}{9 \delta^{2}} c_{2 N-1}-\frac{8 i}{9 \delta^{2}} c_{2 N-3}-q_{22}^{(2)} c_{2 N-1}-q_{21}^{(3)} a_{2 N-2}\right. \\
& +\frac{i u^{*}}{3 \delta} d_{2 N}-\frac{i u^{*}}{3 \delta} d_{2 N-2}+\frac{i|u|^{2}}{8} c_{2 N-1} \\
& \left.+\frac{3 i \delta}{32} d_{2 N}|u|^{2} u^{*}+\frac{u_{t}^{*}}{4} d_{2 N}\right) \text {. }
\end{aligned}
$$

Substituting $u^{\prime}=u\left(a_{2 N} / d_{2 N}\right)-(8 / 3 \delta)\left(b_{2 N-1} / d_{2 N}\right), u^{\prime *}=$ $u^{*}\left(d_{2 N} / a_{2 N}\right)+(8 / 3 \delta)\left(c_{2 N-1} / a_{2 N}\right)$ into $(14 \mathrm{i})$ and $(14 \mathrm{j})$, one can calculate that

$$
\begin{aligned}
& q_{12}^{(1)}=\frac{i u^{\prime}}{3 \delta}+\frac{3 i \delta}{32}\left|u^{\prime}\right|^{2} u^{\prime}-\frac{1}{4} u_{x}^{\prime}, \\
& q_{21}^{(1)}=\frac{i u^{\prime *}}{3 \delta}+\frac{3 i \delta}{32}\left|u^{\prime}\right|^{2} u^{\prime *}-\frac{1}{4} u_{x}^{\prime *} .
\end{aligned}
$$

Summarying the above conclusions, one can arrive at the following theorm.

Theorem 1. The solution q for (1) is mapped into the new solution $q^{\prime}$ under the Darboux transformation:

$$
u^{\prime}=u \frac{a_{2 N}}{d_{2 N}}-\frac{8}{3 \delta} \frac{b_{2 N-1}}{d_{2 N}}, \quad u^{\prime *}=u^{*} \frac{d_{2 N}}{a_{2 N}}+\frac{8}{3 \delta} \frac{c_{2 N-1}}{a_{2 N}} .
$$

\section{Soliton and Breather Solutions for (1)}

In this section, we will construct soliton solutions for (1). Choosing $\phi(\lambda)=\left(\phi_{1}(\lambda), \phi_{2}(\lambda)\right)^{\prime}$ and $\psi(\lambda)=\left(-\psi_{2}^{*}\left(\lambda^{*}\right)\right.$, $\left.\psi_{1}^{*}\left(\lambda^{*}\right)\right)^{\prime}$ as two solutions of the spectral system $(2 \mathrm{a})-(2 \mathrm{~b})$, one can derive that $\lambda_{2 j}=\lambda_{2 j-1}^{*}$ and $\gamma_{2 j}=\gamma_{2 j-1}^{*}$, so $\alpha_{2 j-1}=$ $\left[\phi_{2}\left(\lambda_{2 j-1}\right)-\gamma_{2 j-1} \psi_{2}\left(\lambda_{2 j-1}\right)\right] /\left[\phi_{1}\left(\lambda_{2 j-1}\right)-\gamma_{2 j-1} \psi_{1}\left(\lambda_{2 j-1}\right)\right]$.

For $N=1$, from (5), one can obtain that

$$
\begin{aligned}
& a_{2}=\frac{\lambda_{1}\left|\phi_{2}\right|^{2}+\lambda_{1}^{*}\left|\phi_{1}\right|^{2}}{\left|\lambda_{1}\right|^{2} \lambda_{1}\left|\phi_{1}\right|^{2}+\lambda_{1}^{*}\left|\phi_{2}\right|^{2}}, \\
& b_{1}=\frac{\left(\lambda_{1}^{* 2}-\lambda_{1}^{2}\right) \phi_{1} \phi_{2}^{*}}{\left|\lambda_{1}\right|^{2} \lambda_{1}\left|\phi_{1}\right|^{2}+\lambda_{1}^{*}\left|\phi_{2}\right|^{2}},
\end{aligned}
$$

and $d_{2}=a_{2}^{*}, c_{1}=-b_{1}^{*}$. Then from (16), one can obtain

$$
u^{(1)}=u\left(\frac{\Delta^{*}}{\Delta}\right)^{2}+\frac{8}{3 \delta \Delta^{2}}\left(\lambda_{1}^{2}-\lambda_{1}^{* 2}\right) \phi_{1} \phi_{2}^{*} \Delta^{*},
$$

with $\Delta=\lambda_{1}\left|\phi_{1}\right|^{2}+\lambda_{1}^{*}\left|\phi_{2}\right|^{2}$.

For $N=2$, from $d_{4}=a_{4}^{*}, c_{3}=-b_{3}^{*}$, and (5), one can obtain that

$$
\begin{gathered}
\sum_{n=1}^{2}\left(a_{2 n} \lambda_{2 j-1}+\alpha_{2 j-1} b_{2 n-1}\right) \lambda_{2 j-1}^{2 n-1}=1, \\
\sum_{n=1}^{2}\left(a_{2 n} \lambda_{2 j-1}^{*} \alpha_{2 j-1}^{*}-b_{2 n-1}\right) \lambda_{2 j-1}^{*(2 n-1)}=-\alpha_{2 j-1},
\end{gathered}
$$

with $j=1,2$, so (19a)-(19b) result:

$$
a_{4}=\frac{\Omega_{a_{4}}}{\Omega}, \quad b_{3}=\frac{\Omega_{b_{3}}}{\Omega},
$$

where

$$
\Omega=\left|\begin{array}{cccc}
\lambda_{1}^{2} & \lambda_{1} \alpha_{1} & \lambda_{1}^{4} & \alpha_{1} \lambda_{1}^{3} \\
\lambda_{3}^{2} & \lambda_{3} \alpha_{3} & \lambda_{3}^{4} & \alpha_{3} \lambda_{3}^{3} \\
\lambda_{1}^{* 2} \alpha_{1}^{*} & -\lambda_{1}^{*} & \lambda_{1}^{* 4} \alpha_{1}^{*} & -\lambda_{1}^{* 3} \\
\lambda_{1}^{* 2} \alpha_{3}^{*} & -\lambda_{3}^{*} & \lambda_{3}^{* 4} \alpha_{3}^{*} & -\lambda_{3}^{* 3}
\end{array}\right| .
$$

$\Omega_{a_{4}}$ and $\Omega_{b_{3}}$ are produced from $\Omega$ by replacing its third and fourth columns with $\left(1,1,-\alpha_{1}^{*},-\alpha_{3}^{*}\right)$, respectively. Then the two-soliton solutions for (1) can be derived as

$$
u^{(2)}=u \frac{a_{4}}{d_{4}}-\frac{8}{3 \delta} \frac{b_{3}}{d_{4}} .
$$

Then we we mainly discuss soliton solutions from three different seeds. 
Case 1. Considering the vanishing background of $u=0$, we can generate elastic interactions and bound states between two solitons for (1) as shown in Figure 1.

Figure 1(a) depicts the interactions of two solitons on the vanishing background. One can find that main features of the interaction are that the shapes, amplitudes, and pulse widths all remain invariant except for slightly visible phase shifts, so the interaction is elastic. Figure 1(b) shows that when suitable parameters are chosen, two bound solitons with the same amplitude propagate in parallel without any effect on each other even if the propagation distance grows long enough. From Figure 1, we can conclude that the parallel bound solitons will form when $\left|\lambda_{1}\right| \rightarrow\left|\lambda_{2}\right|$, while if the value of ||$\lambda_{1}|-| \lambda_{2}||$ increases, the elastic interactions between two solitons will happen.

Case 2. In the case of the cw background as $u=u_{c} \exp i(\kappa t+$ $\omega z)$, we can derive the nonlinear dispersion relation $\omega=$ $\left(4 u_{c}^{2}-8 \kappa^{2}+3 u_{c}^{2} \kappa \delta+16 \Gamma\right) / 16$ for (1). By the method of separation of variables and the superposition principle, we derive

$$
\phi_{1}=\left(c_{1} e^{\theta_{1}}+c_{2} e^{\theta_{2}}\right) \exp i(\kappa t+\omega z), \quad \phi_{2}=c_{3} e^{\theta_{1}}+c_{4} e^{\theta_{2}},
$$

where

$$
\begin{aligned}
& \theta_{1}=\frac{t}{2}\left(-i \kappa-\frac{1}{3 \delta} \sqrt{B^{2}-A^{2}}\right)+\frac{z}{2}\left(-i \omega-\frac{1}{3 \delta} \sqrt{B^{2}-A^{2}} \Theta\right), \\
& \theta_{2}=\frac{t}{2}\left(-i \kappa+\frac{1}{3 \delta} \sqrt{B^{2}-A^{2}}\right)+\frac{z}{2}\left(-i \omega+\frac{1}{3 \delta} \sqrt{B^{2}-A^{2}} \Theta\right),
\end{aligned}
$$

with

$$
\begin{gathered}
A=4\left(\lambda_{1}^{2}-1\right), \quad B=3 i u_{c} \delta \lambda_{1}, \\
\Theta=\frac{9 \delta^{2} u_{c}^{2}-32\left(\lambda_{1}^{2}-1\right)}{48 \delta} .
\end{gathered}
$$

For simplicity, setting $c_{4}=c_{1}$ and through direct computations, we can generate that

$$
c_{3}=\frac{\theta_{1 t}+i \kappa-\gamma}{(i / 2) \lambda_{1} u_{c}} c_{1}, \quad c_{2}=\frac{(i / 2) \lambda_{1} u_{c}}{\theta_{1 t}+i \kappa-\gamma} c_{4},
$$

with $\gamma=2 i\left(\lambda_{1}^{2}-1\right) / 3 \delta$. Substituting (27) into (18) and taking $\operatorname{Re}\left(\lambda_{1}\right)=\operatorname{Im}\left(\lambda_{1}\right), \kappa=0$, we can obtain Akhmedievbreather solutions on the nonvanishing background for (1) as shown in Figure 2(a). One can observe that the main feature is propagation of the Akhmediev breather which is periodic in the space coordinate and aperiodic in the time coordinate. Therefore, it is considered as a modulation instability (MI) process in which a cw beam becomes unstable [15]. MI was predicted to occur in optical fibers and was experimentally observed [16].

Iterating the DT again, we can obtain the two-breather solution for (1) as shown in Figures 2(b), 3(a), and 3(b). One can observe that the interactions between those breathers are also elastic. Through adjusting the value of $\lambda_{1}$ and $\lambda_{2}$, we can control the directions of those breathers as shown in those figures.

Case 3. Considering the constant background of $u=\kappa_{1}+i \kappa_{2}$, (1) gives the relation $\kappa_{1}^{2}+\kappa_{2}^{2}=-4 \Gamma$. Defining $\lambda=A_{c}+i \kappa_{c}$, and for simplicity setting $\kappa_{c}=\sqrt{8\left(A_{c}^{2}-1\right)-9 \Gamma \delta^{2}}$, we can obtain

$$
\phi_{1}=c_{1} e^{\vartheta}+c_{2} e^{-\vartheta}, \quad \phi_{2}=c_{3} e^{\vartheta}+c_{4} e^{-\vartheta}
$$

where

$$
\begin{gathered}
\vartheta=2 t\left(\xi_{1}+i \eta_{1}\right)+z\left(\xi_{1}+i \eta_{1}\right) \frac{8\left(\lambda^{2}-1\right)+9 \Gamma \delta^{2}}{36 \delta^{2}}, \\
c_{3}=\frac{\vartheta_{t}-(2 i / 3 \delta)\left(\lambda^{2}-1\right)}{(i / 2) \lambda\left(\kappa_{1}+i \kappa_{2}\right)} c_{1}, \\
c_{2}=\frac{(i / 2) \lambda\left(\kappa_{1}+i \kappa_{2}\right)}{-\vartheta_{t}-(2 i / 3 \delta)\left(\lambda^{2}-1\right)} c_{4},
\end{gathered}
$$

with

$$
\xi_{1}+i \eta_{1}=\sqrt{\beta(16+\beta)}, \quad \beta=9 \Gamma A_{c}^{2} .
$$

Owing to $\beta$ which is always negative, we discuss two different cases according to the sign of $(16+\beta)$.

(A) When $(16+\beta)>0$, the value of $\beta(16+\beta)$ will be negative; then we can arrive at $\xi_{1}=0, \eta_{1}=$ $\sqrt{-\beta(16+\beta)}$. Substituting these conclusions and (27) into (18), we can derive the Ma-breather solution for (1) as shown in Figure 4(a).

(B) When $(16+\beta)<0$, the value of $\beta(16+\beta)$ will be positive; then we can arrive at $\xi_{1}=\sqrt{\beta(16+\beta)}, \eta_{1}=$ 0 . Substituting these conclusions and (27) into (18), we can derive the Akhmediev-breather solution for (1) as shown in Figure 4(b).

One can observe from Figure 4(a) that the breathers time periodically propagate on the constant backgrounds; that is, they are the Ma-breathers. In addition, as $\kappa_{1}+i \kappa_{2}$ approaches zero, the Ma-breather will become the one-soliton solution. Since $\kappa_{1}^{2}+\kappa_{2}^{2}=-4 \Gamma$ and $\Gamma$ denotes the gain or loss coefficient in in (1), we can conclude that the absence of the gain or loss term can become breathers into one-soliton solutions.

From Figure 4(b), one can find that the Akhmediev breather is periodic in the space coordinate and aperiodic in the time coordinate. Generally, the time-aperiodic solution can be regarded as a homoclinic or separatrix trajectory in the infinite-dimension phase space of the solutions for (1) with periodic boundary conditions in space. Through numerical simulation, one can gain the facts that are in Figure 4(b) as follows:

(1) the periods are in inverse proportion to the value of $16+\beta$, so the group velocities of the Akhmediev breathers are dependent on parameters $\beta$;

(2) parameters $A_{c}$ can affect the amplitudes. 


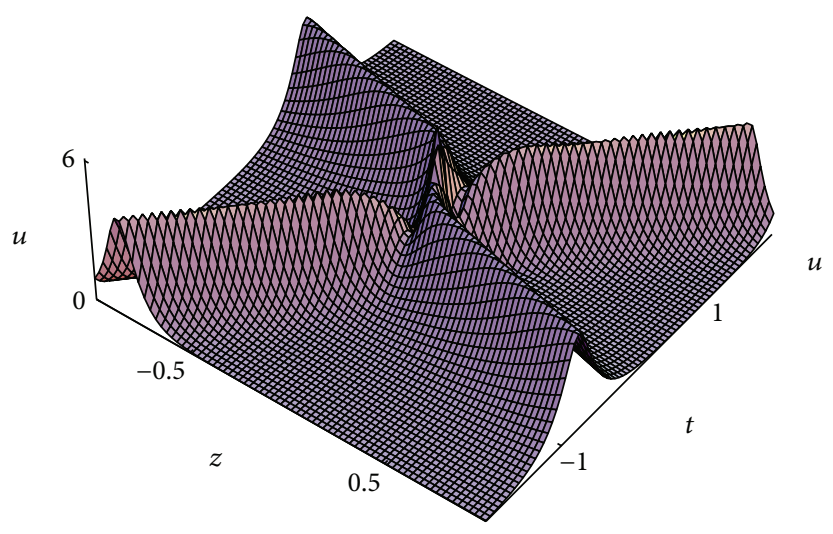

(a)

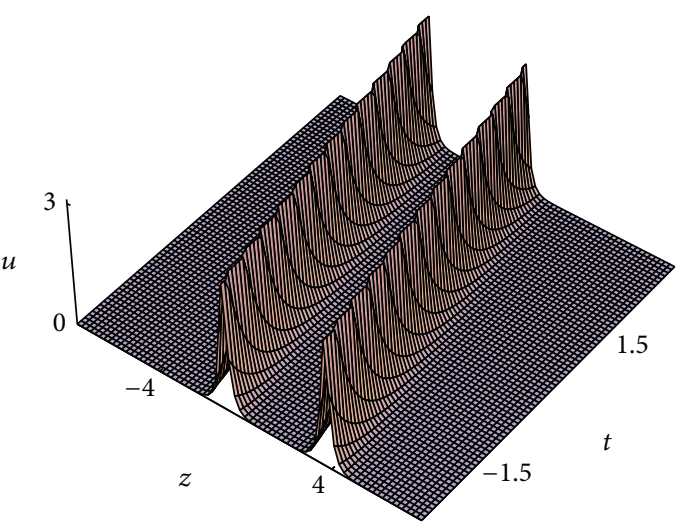

(b)

Figure 1: Evolution of the two-soliton solutions of (1). Parameters are (a) $\lambda_{1}=2+2 i, \lambda_{2}=3+2 i, \delta=2.5, \Gamma=0.1$ and (b) $\lambda_{1}=1+2 i$, $\lambda_{2}=1.0001-2 i, \delta=2.5, \Gamma=0.1$.

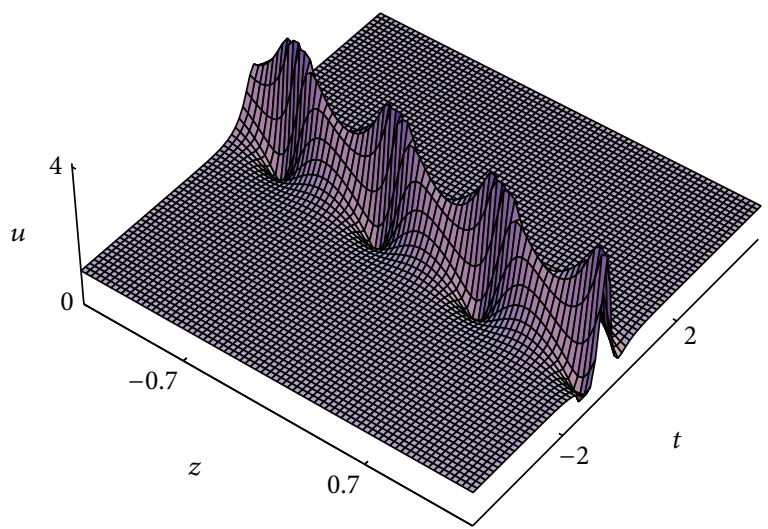

(a)

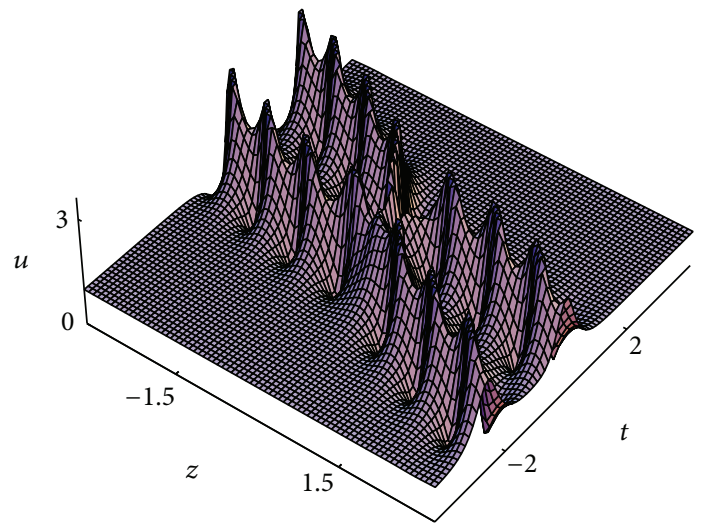

(b)

Figure 2: Evolution of the breather solutions of (1). Parameters are (a) $u_{c}=1, \kappa=0, \delta=3, \lambda_{1}=2.05+2.05 i, \Gamma=0$ and (b) $u_{c}=1, \kappa=0$, $\lambda_{1}=2.05+2.05 i, \lambda_{2}=2+2 i, \delta=3, \Gamma=0$.

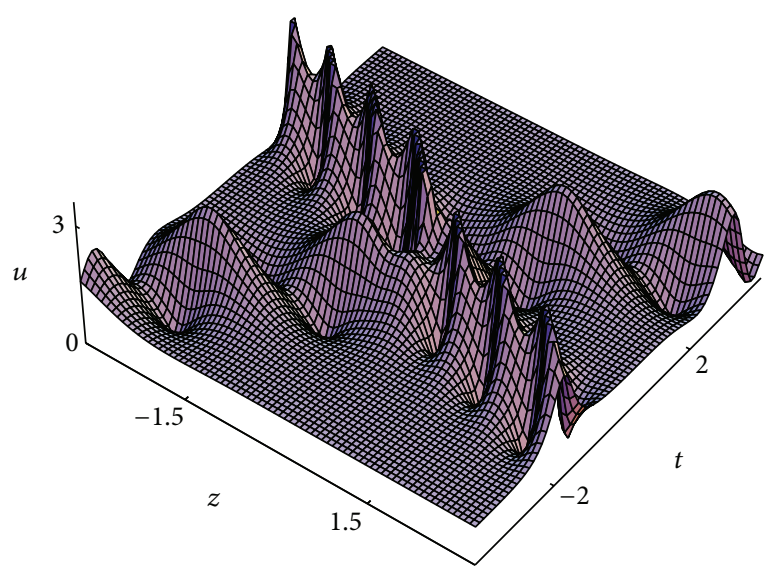

(a)

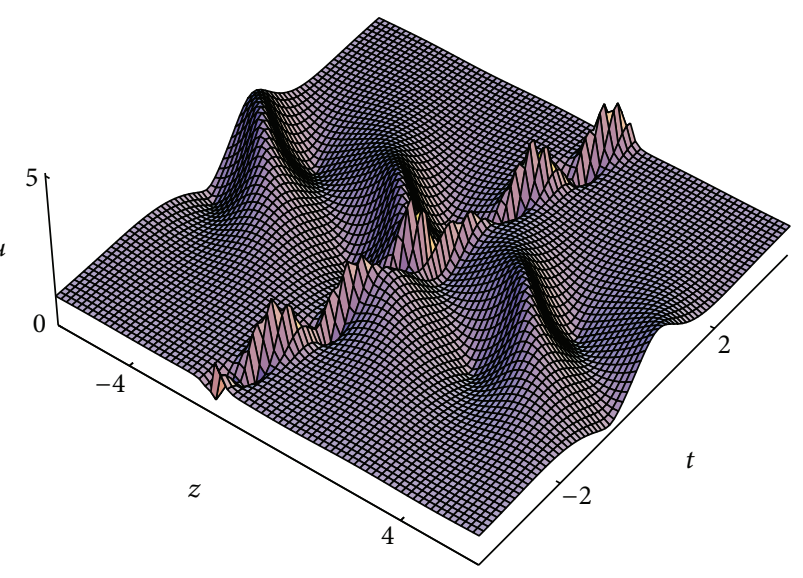

(b)

FIgURE 3: Evolution of the breather solutions of (1). Parameters are (a) $u_{c}=1, \kappa=0, \lambda_{1}=2.05+1.05 i, \lambda_{2}=2+2 i, \delta=3, \Gamma=0$ and (b) $u_{c}=1$, $\kappa=0, \lambda_{1}=1.5+i, \lambda_{2}=3+1.1 i, \delta=3, \Gamma=0$. 


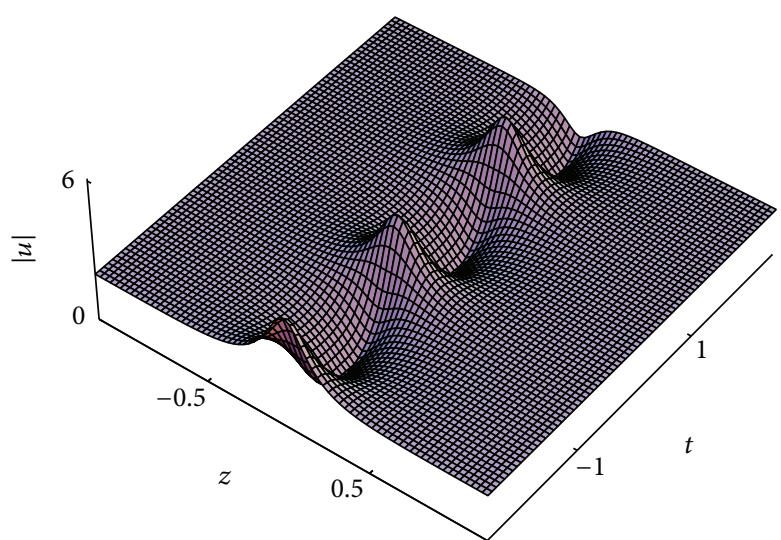

(a)

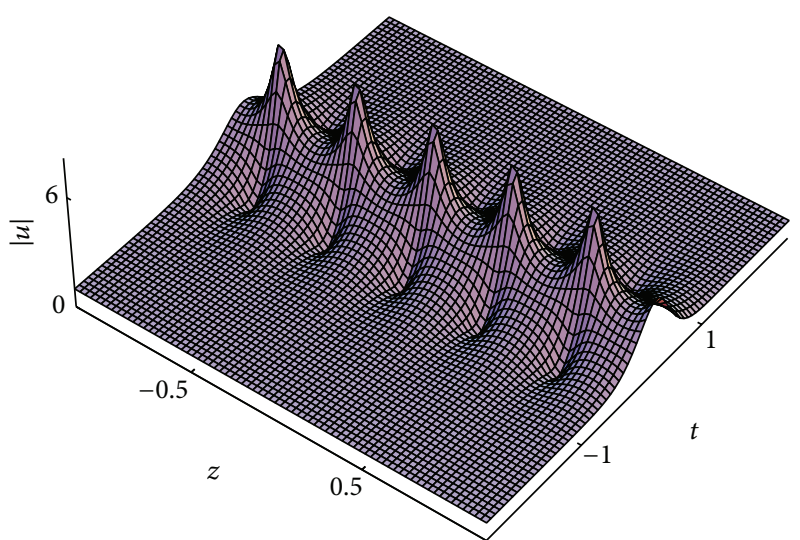

(b)

FIgURE 4: Evolution of the breather solutions of (1). Parameters are (a) $A_{c}=0.5, \kappa_{1}=1, \kappa_{2}=1, \delta=1$ and $(\mathrm{b}) A_{c}=0.05, \kappa_{1}=1, \kappa_{2}=1$, $\delta=1$.

\section{Conclusions}

Our attention has been focused on (1) which describes the propagation of the subpicosecond or femtosecond optical pulse in a monomodal optical fiber. With symbolic computation, we have constructed $N$-fold Darboux transformation and derived two-soliton and breather solutions on the vanishing and two types of nonvanishing backgrounds, respectively. In addition, some figures have been plotted to display the dynamic characteristics of those solitons.

\section{Conflict of Interests}

The authors declare that there is no conflict of interests regarding the publication of this paper.

\section{Acknowledgments}

The authors express sincere thanks to each member of their discussion group for their suggestions. This work has been supported by the Special Funds of the National Natural Science Foundation of China under Grant nos. 11347165 and 11172194, by the Natural Science Foundation of Shanxi Province under Grant no. 2012021011-3, and by the Science and Technology Innovation Fund of Taiyuan University of Technology.

\section{References}

[1] A. Biswas and S. Konar, "Quasi-particle theory of optical soliton interaction," Communications in Nonlinear Science and Numerical Simulation, vol. 12, no. 7, pp. 1202-1228, 2007.

[2] R. Kohl, A. Biswas, D. Milovic, and E. Zerrad, "Optical soliton perturbation in a non-Kerr law media," Optics and Laser Technology, vol. 40, no. 4, pp. 647-662, 2008.

[3] P. Green, D. Milovic, A. K. Sarma, D. A. Lott, and A. Biswas, "Dynamics of super-sech solitons in optical fibers," Journal of
Nonlinear Optical Physics and Materials, vol. 19, no. 2, pp. 339370, 2010.

[4] L. Girgis, D. Milovic, S. Konar, A. Yildirim, H. Jafari, and A. Biswas, "Optical Gaussons in birefringent fibers and DWDM systems with intermodal dispersion," Romanian Reports in Physics, vol. 64, no. 3, pp. 663-671, 2012.

[5] A. Biswas, M. Fessak, S. Johnson et al., "Optical soliton perturbation in non-Kerr law media: traveling wave solution," Optics and Laser Technology, vol. 44, no. 1, pp. 263-268, 2012.

[6] M. Savescu, K. R. Khan, R. W. Kohl, L. Moraru, A. Yildirim, and A. Biswas, "Optical soliton perturbation with improved nonlinear schrödinger's equation in nano fibers," Journal of Nanoelectronics and Optoelectronics, vol. 8, no. 2, pp. 208-220, 2013.

[7] G. P. Agrawal, Nonlinear Fiber Optics, Academic Press, San Diego, Calif, USA, 2001.

[8] O. C. Wright, "Homoclinic connections of unstable plane waves of the modified nonlinear Schrödinger equation," Chaos, Solitons and Fractals, vol. 20, no. 4, pp. 735-749, 2004.

[9] Z. Y. Chen and N. N. Huang, "Explicit N-soliton solution of the modified nonlinear Schrödinger equation," Physical Review A, vol. 41, p. 4066, 1990.

[10] S. L. Liu and W. Z. Wang, "Exact $N$-soliton solution of the modified nonlinear Schrödinger equation," Physical Review E, vol. 48, p. 3054, 1993.

[11] C. H. Gu, H. S. He, and Z. X. Zhou, Darboux Transformation in Soliton Theory and Its Geometric Applications, Shanghai Scientific and Technical, Shanghai, China, 2005.

[12] V. B. Matveev and M. A. Sall', "Scattering of solitons in the formalism of the Darboux transform," Journal of Soviet Mathematics, vol. 34, no. 5, pp. 1983-1987, 1986.

[13] R. Guo and H.-Q. Hao, "Breathers and localized solitons for the Hirota-Maxwell-Bloch system on constant backgrounds in erbium doped fibers," Annals of Physics, vol. 344, pp. 10-16, 2014.

[14] R. Guo and H.-Q. Hao, "Breathers and multi-soliton solutions for the higher-order generalized nonlinear Schrödinger equation," Communications in Nonlinear Science and Numerical Simulation, vol. 18, no. 9, pp. 2426-2435, 2013. 
[15] B. K. Som, M. R. Gupta, and B. Dasgupta, "Reduction of the Boussinesq type of equation to modified Hirota equation," Journal of the Physical Society of Japan, vol. 47, no. 4, pp. 12961298, 1979.

[16] K. Tai, A. Tomita, and A. Hasegawa, "Observation of modulational instability in optical fibers," Physical Review Letters, vol. 56, p. $135,1986$. 


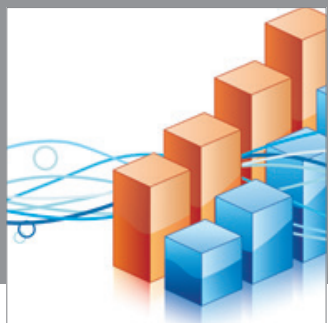

Advances in

Operations Research

mansans

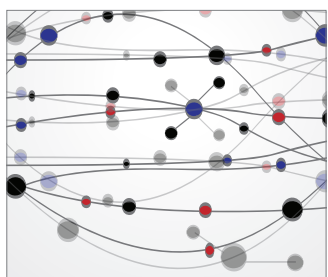

The Scientific World Journal
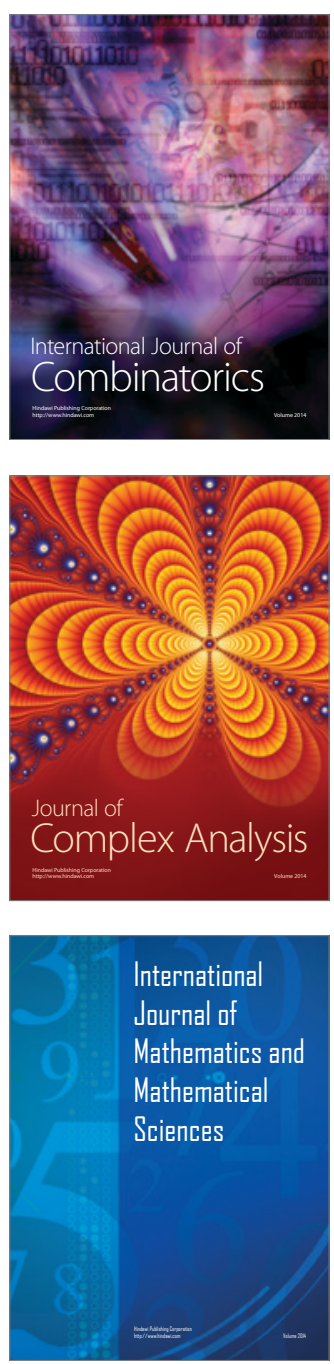
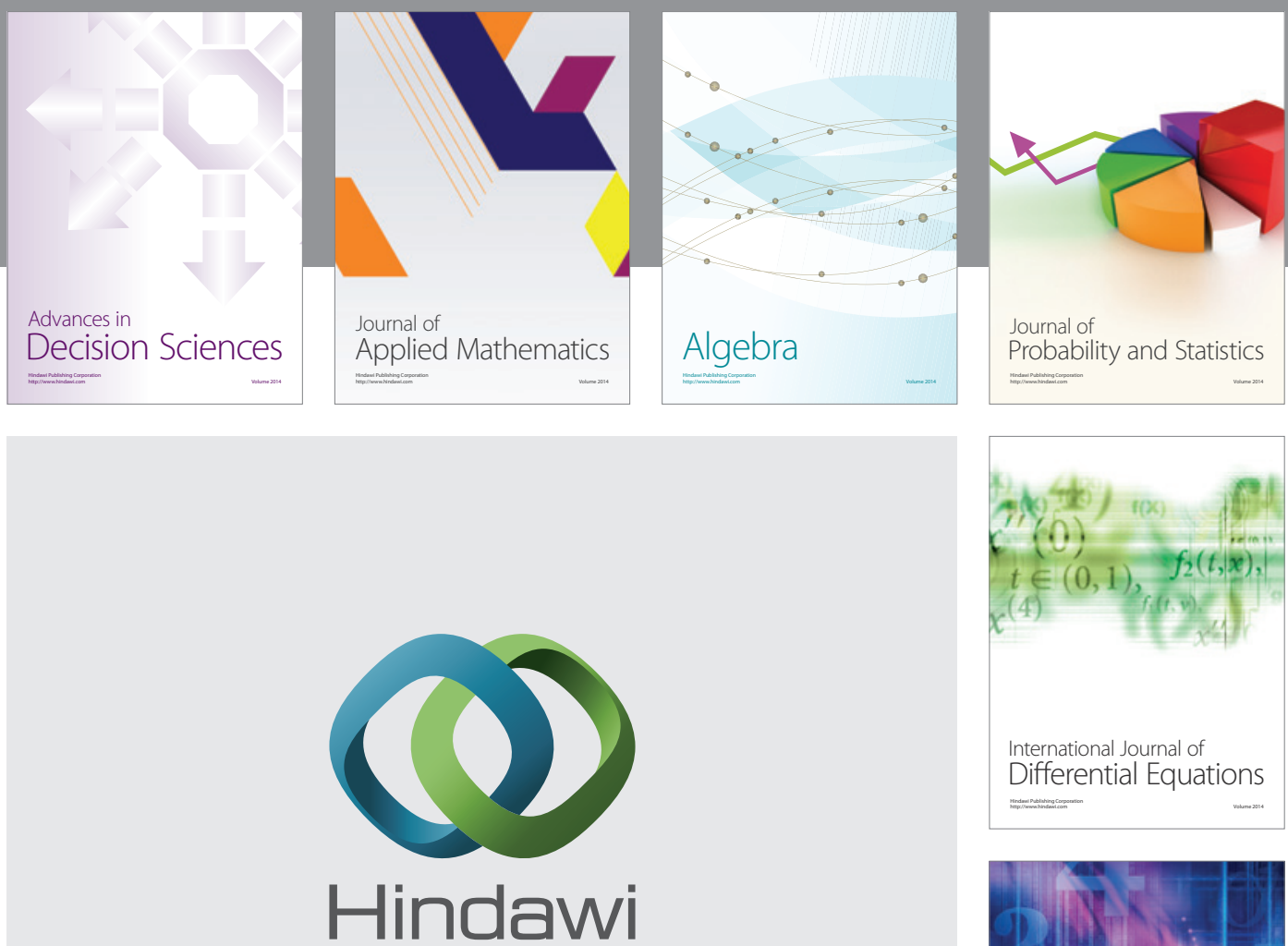

Submit your manuscripts at http://www.hindawi.com
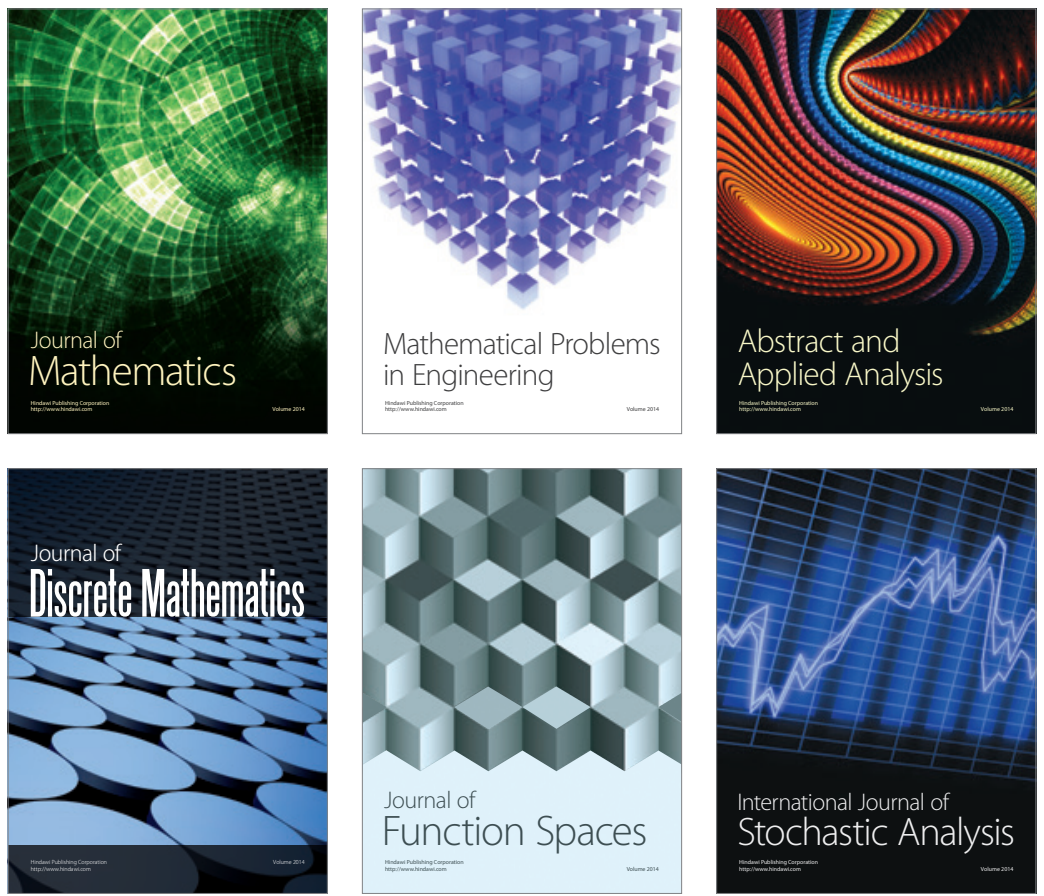

Journal of

Function Spaces

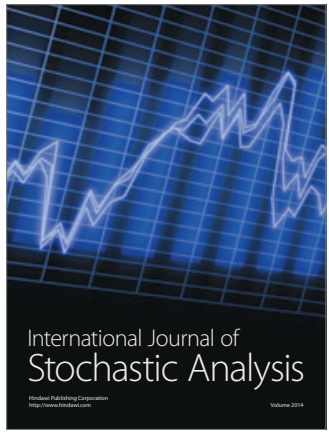

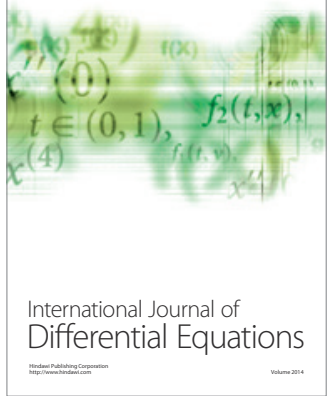
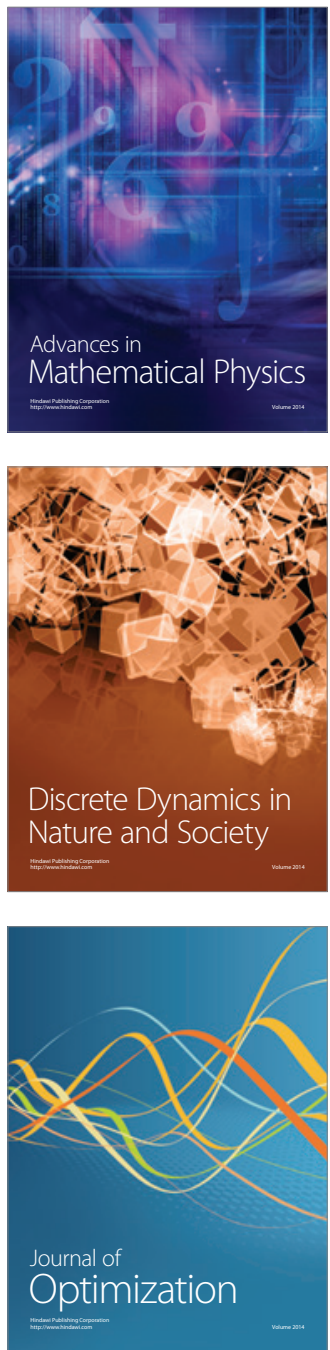